\title{
ACEPTACIÓN TECNOLÓGICA DE LA PUBLICIDAD EN DISPOSITIVOS MÓVILES EN COLOMBIA*
}

\author{
Recibido: 15 de febrero de 2014 Aprobado 19 de agosto 2015
}

\author{
Bernardo Ballesteros Díaz ${ }^{* *}$ \\ Juan Fernando Tavera Mesías ${ }^{* * *}$ \\ Daniela Castaño Serna ${ }^{* * *}$
}

\section{RESUMEN}

Este artículo analiza el comportamiento de aceptación tecnológica de la publicidad móvil en Colombia, utilizando el modelo TAM (Technology Acceptance Model) y adicionando el concepto de confianza como variable que influye en la relación entre anunciantes y receptores de la publicidad móvil. El modelo planteado fue contrastado mediante una muestra empírica de 530 individuos, se comprobó la fiabilidad y validez del mismo y se validaron las hipótesis mediante ecuaciones estructurales. Para el caso de los consumidores colombianos, los resultados demuestran el ajuste del modelo y confirman que la confianza influye directamente en la intención de uso; sin embargo, la utilidad percibida se constituye como el principal determinante del uso de la publicidad móvil.

\section{PALABRAS CLAVE}

Publicidad, Dispositivos móviles, Aceptación tecnológica, TAM, Marketing.

\section{CLASIFICACIÓN JEL}

M31, M37

\section{CONTENIDO}

Introducción, 1. Modelo de Aceptación Tecnológica, 2. La confianza en la publicidad móvil, 3. Modelo propuesto, 4. Metodología, 5. Resultados, 6. Conclusiones, Bibliografía.

\footnotetext{
Artículo de investigación, producto del proyecto "Aceptación de la Publicidad móvil en Colombia", adscrito al Grupo de investigación en Marketing -iMARK. Facultad de Ciencias Económicas, Universidad de Antioquia, Clasificación B Colciencias. Proyecto ejecutado en el período: 2012-2013.

* Magíster en Administración, Universidad EAFIT, Medellín-Colombia. Especialista en Gerencia, Universidad Pontificia Bolivariana, Medellín- Colombia. Administrador de Empresas, Universidad Nacional, Manizales- Colombia. Profesor tiempo completo Universidad de Antioquia, Facultad de Ciencias Económicas- Departamento Ciencias Administrativas, Medellín, Colombia. Investigador Grupo de Investigación en Marketing iMARK., Teléfono: (+57 4) 219 5839. Celular (+57) 30065446 63. Calle 70 N. 52 - 21 Bloque 13 Of. 103. Correo electrónico: bballesteros@ economicas.udea.edu.co.

... Candidato, Doctorado en Marketing, Universidad de Valencia, Valencia - España. Master Oficial en Marketing e Inv. de Mercados, Universidad De Valencia-España. Magíster en Administración, Universidad EAFIT, Medellín- Colombia. Magíster en Administración, Universidad EAFIT. Economista, Universidad Pontificia Bolivariana, MedellínColombia. Profesor vinculado Universidad de Antioquia, Facultad de Ciencias Económicas- Departamento Ciencias Administrativas, Medellín, Colombia. Investigador Grupo de Investigación en Marketing iMARK. Teléfono: (+57 4) 219 8821. Celular (+57) 30173992 39- Calle 70 N. 52 - 21 Bloque 13 Oficina 105. Correo electrónico: jftavera@ economicas.udea.edu.co

...* Estudiante, Maestría en Gestión de la Ciencia, la Tecnología y la Innovación, Universidad de Antioquia, MedellínColombia. Profesional en Negocios Internacionales, Fundación Universitaria Luis Amigó, Medellín-Colombia. Docente de catedra Universidad de Antioquia, Facultad de Ciencias Económicas- Departamento Ciencias Administrativas, Medellín, Colombia. Consultora Master Grupo de Investigación en Marketing iMARK. Teléfono: $(+57$ 4) 219 2075. Celular (+57) 3005579747 Calle 65 \# 55 - 46. Correo electrónico: daniela.castanos@udea.edu.co.
} 


\section{TECHNOLOGICAL ACCEPTANCE OF ADVERTISING IN MOBILE DEVICES IN COLOMBIA}

\section{ABSTRACT}

This article looks for analyzing the behavior of technological acceptance of advertising in mobile devices in Colombia, using TAM model (Technology Acceptance Model) and adding the confidence as a variable which influences in the relation between advertisers and sectors or mobile advertising. The model was contrasted through an empirical sample of 530 individuals; reliability and validity of the model was verified and hypothesis were validated by means of structural equations. In the case of Colombian consumers, results show the adjustment of the model and confirm that the confidence directly influences in the use intention; however, the usefulness perceived, constitutes as the main dewterminant of use of mobile advertising.

\section{KEY WORDS}

Advertising, Mobile devices, Technological acceptance, TAM, Marketing.

\section{JEL CLASSIFICATION}

M31, M37

\section{CONTENT}

Introduction 1. Technological Acceptance Model 2. Confidence in mobile advertising 3. Proposed model 4. Methodology 5. Results 6. Conclusions, Bibliography.

\section{ACEITAÇÃO TECNOLÓGICA DA PUBLICIDADE EM DISPOSITIVOS MÓVEL NA COLÔMBIA \\ RESUMO}

O presente artigo procura analisar o comportamento tecnológico da aceitação da publicidade móvel na Colômbia, usando o modelo TAM (Tecnologia aceitação Modelo) e adicionar o conceito de confiança como uma variável que afeta as relações entre os anunciantes e os destinatários da publicidade móvel. O modelo foi calibrado através de uma amostragem empírica de 530 indivíduos, foi encontrada a confiabilidade e a validade do mesmo, validando as hipóteses usando equações estruturais. No caso dos consumidores colombianos, os resultados mostram o ajuste do modelo e confirmar que o confiança influencia diretamente na intenção de utilizar, no entanto, a utilidade se constitui como a principal determinante da utilização de publicidade móvel.

\section{PALAVRAS-CHAVE}

Publicidade, dispositivos móveis, tecnologia aceitação, TAM, Marketing.

\section{CLASSIFICAÇÃO JEL}

M31, M37

\section{CONTEÚDO}

Introdução 1. Aceitação Tecnologia Modelo 2. Confiança na publicidade móvel, 3. Modelo proposto, 4. Metodologia 5. Os resultados, 6. Conclusões, Bibliografia 


\section{INTRODUCCIÓN}

Los medios de comunicación tradicionales son los más utilizados para transmitir la publicidad a los consumidores. Sin embargo, la dinámica de las tecnologías de información y comunicación (TIC), tal y como lo plantean Lamb, Hair y Mcdaniel (1998) permite nuevas formas de anunciar a través de Internet, dispositivos electrónicos y móviles que ofrecen ventajas en la comunicación inmediata y directa con el usuario, representando un enorme desafío para los anunciantes, puesto que los consumidores tienen más control sobre la relación de mercadeo que la que han tenido con los medios tradicionales.

Por esta razón, las nuevas estrategias de comunicación utilizan la publicidad móvil como una forma de marketing electrónico que, a través de medios publicitarios, permite interactuar de forma directa con los consumidores, busca una respuesta directa de estos y, además, se convierte en una forma de marketing móvil que promueve la venta y provisión de información de productos o servicios (Bamba y Barnes, 2007) y agrega valor al enviar mensajes publicitarios que pueden estar contextualizados con el lugar, las necesidades del momento y el dispositivo que están usando (Yuan y Tsao, 2003).

En Colombia, de acuerdo con el MTIC (2015), al término del primer trimestre de 2015, el número absoluto de abonados en servicio de telefonía móvil en Colombia alcanzó un total de 56.139.892, presentando un aumento de 8,2 puntos porcentuales con relación al índice de penetración del mismo trimestre del año anterior, el cual se ubicó en el 108,3 \% y según el reporte de IAB Colombia (2015) la inversión en medios digitales móviles ha tenido un crecimiento de 117 \% del cuarto trimestre de 2013 al cuarto trimestre de 2014. Estas cifras representan un potencial para el desarrollo de la publicidad móvil en el país.

Colombia, como país en desarrollo, presenta bajos índices de penetración de la tecnología y, en consecuencia, condiciones especiales de aceptación tecnológica, en comparación con mercados desarrollados. Al respecto, existen estudios académicos aplicados a estos últimos mercados, que han trabajado la publicidad móvil a través del modelo de aceptación tecnológica para determinar el comportamiento de los usuarios (p. ej. He y Lu (2007); Zhang y Mao (2008); Barakat y Sheikh, (2010); Bart, Stephen y Sarvary (2014); Sanz-Blas, Ruiz- Mafé y Martí-Parreño (2015). Para complementar la literatura académica en Colombia, que es limitada sobre el tema, interesa conocer cuál es la aceptación tecnológica de la publicidad a través de dispositivos móviles. 
El objetivo del presente artículo es analizar los antecedentes de la aceptación tecnológica de la publicidad en dispositivos móviles en Colombia, haciendo uso del modelo TAM (Technology Acceptance Model) planteado por Davis (1989), a partir de la revisión de los antecedentes relevantes de la intención de uso de la publicidad en dispositivos móviles, que brinde información sobre el comportamiento de los consumidores en el país. El objetivo requiere modelar dicho comportamiento apoyándose en el TAM y la variable confianza utilizada en otros estudios en el contexto mundial (Barakat y Sheikh (2010); Ba (2001); Molina, López, y Soto (2012)). Esta variable es un determinante fundamental en la intención y el uso de la publicidad móvil, por la separación espacial entre anunciantes y receptores, siendo la incertidumbre un aspecto relevante dentro de esta relación (Ba, 2001). Para el análisis se presentan siete hipótesis, cada una validada por algunas escalas de medición, utilizadas en estudios similares sobre la temática (Davis (1989); Moore y Benbasat (1991); Taylor y Todd (1995); Kim, Ferrini y Rao (2008); Kim, Chung y Lee (2011); Taylor y Todd (1995); Coyle y Thorson (2001), Kim y Biocca (1997)) y se contrastaron las hipótesis con una muestra empírica de datos a través de modelos de ecuaciones estructurales.

En el presente artículo se hace una revisión del marco teórico y estado del arte relacionado con los antecedentes de la aceptación tecnológica de la publicidad en dispositivos móviles y a partir de esta información se plantea un modelo de hipótesis para el caso Colombiano; se explica la metodología utilizada para contrastar con una muestra empírica de datos, se analizan los resultados y se presentan conclusiones que permiten afirmar que la Utilidad percibida es el principal antecedente que explica el comportamiento de aceptación por parte de los consumidores, según el modelo propuesto.

\section{MODELO DE ACEPTACIÓN TECNOLÓGICA}

El modelo de aceptación tecnológica (TAM) presentado por Davis (1989), es una adaptación al modelo TRA (teoría de acción razonada) presentado por Fishbein y Ajzen (1975), para predecir e identificar los comportamientos de los usuarios frente a las tecnologías, facilitar su comprensión y generar posibles acciones, teniendo en cuenta determinantes afectivos y cognitivos, y considerando factores internos y externos tales como creencias, actitudes e intenciones, que interactúan con los sistemas y definen el comportamiento futuro de los usuarios.

El TAM presenta dos variables: la utilidad percibida y la facilidad de uso percibida como los antecedentes de los usuarios hacia la actitud y hacia la intención de uso de las tecnologías. La utilidad percibida es el grado en que una persona cree que el uso de un sistema en particular mejora su desempeño en el trabajo y la facilidad 
de uso percibida es el grado en que una persona cree que el uso de un sistema en particular estaría libre de esfuerzos (Davis, 1989).

Según el modelo, la intención es el principal determinante del uso de las tecnologías y está influenciada por la actitud de los usuarios resultante de los efectos positivos que se presentan en las percepciones y experiencias de estos frente a la facilidad de uso y utilidad percibidas.

El TAM se sustenta en las variables intención de uso y actitud, que son tratadas en el TRA (Ajzen y Fishbein, 1977) como influyentes en el comportamiento de las personas según estudios basados en la psicología social y que en este modelo se orienta a determinar la intención de uso de la tecnología. Se refiere a la intención, como la actitud previa al comportamiento que determina su ejecución (Fishbein y Ajzen (1975); Yang, Liu y Zhou (2012)). Por su parte, la actitud se refiere al grado en que una persona tiene una conducta favorable o desfavorable en una cuestión (Ajzen y Fishbein (1977); Davis, Bagozzi y Warshaw (1989)).

La evolución del TAM se ha concentrado en explicar antecedentes de las percepciones de utilidad y facilidad de uso. Venkatesh y Davis (2000) proponen el TAM2 como una extensión al tradicional, en la que se concentran diferentes antecedentes que influencian la utilidad percibida, como lo son la norma subjetiva proveniente de la TRA (Fishbein y Ajzen, 1975), la experiencia, la voluntad, la imagen, la relevancia del trabajo, la calidad del resultado de la tecnología, y la capacidad de demostración.

Por su parte, Venkatesh y Bala (2003) proponen el TAM3 como un avance adicional concentrado hacia las intervenciones, en el que adicionan antecedentes para la utilidad percibida provenientes del TAM2 e incluyen antecedentes de la facilidad de uso percibida, tales como la auto-eficacia con los computadores, la percepción de control externo, la aprehensión hacia los computadores, el playfulness con los computadores (espontaneidad cognitiva en las interacciones con estos), la usabilidad objetiva y el entretenimiento percibido. Para ambos modelos, el TAM2 y el TAM3, se excluye la actitud y se entablan relaciones directas desde las percepciones de facilidad de uso y utilidad hacia la intención de comportamiento de aceptación de la tecnología.

Estas evoluciones pueden ser aplicadas al comportamiento del consumidor; sin embargo, la investigación presente, más que enfocarse en explicar los antecedentes generales del TAM, incluyó variables complementarias propias del contexto y objeto de estudio, como la confianza, por lo que fue pertinente mantener el TAM tradicional. 
El modelo TAM se ha utilizado en diferentes contextos y temáticas relacionadas tales como: adopción e intención de consumo de sistemas electrónicos e Internet móvil (p. ej, Davis, Bagozzi y Warshaw (1989); Lin, Shih, y Sher (2007)), aceptación de los consumidores de las innovaciones tecnológicas y de servicios móviles avanzados (p. ej. Bina, Karaiskos y Giaglis (2007); Lopez, Molina y Bouwman, (2008); Hsiao y Chang (2014); Muk y Chung (2015)), adopción y gestión de relaciones con clientes del comercio móvil (p. ej. Chong, Chan y Ooi (2012); Jun y Lee (2007)) intenciones de los consumidores a actualizar los teléfonos móviles (p. ej. Tseng y Lo (2011)), el papel del marketing en el comercio móvil (p. ej. Jun y Lee, (2007a)) y las actitudes de los consumidores hacia la publicidad móvil (p. ej. Altuna y Konuk (2009); Zhang y Mao (2008)). La figura 1 representa gráficamente la relación entre las variables del modelo TAM tal como se señaló.

Figura 1. Modelo TAM

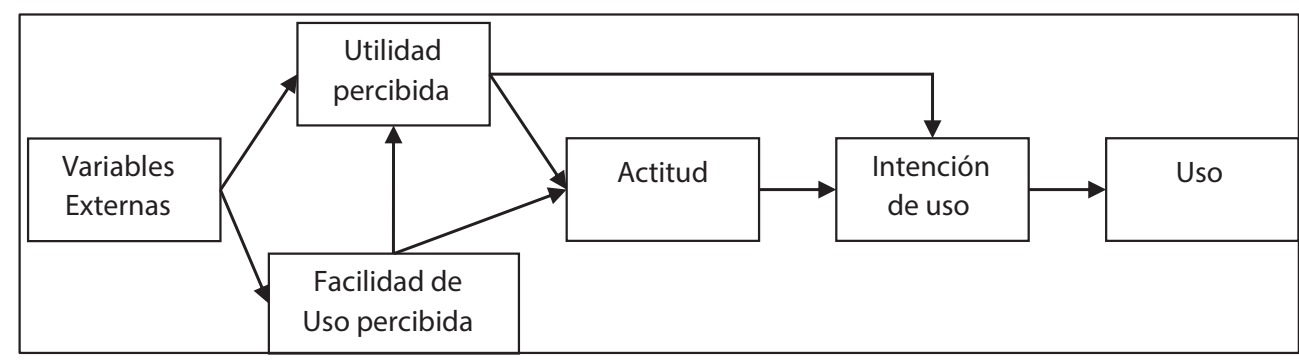

Fuente: Davis, Bagozzi y Warshaw (1989)

A continuación se proponen las hipótesis trabajadas de acuerdo con el modelo TAM:

H1: Facilidad de uso percibida influye positivamente sobre la utilidad percibida

H2: Facilidad de uso percibida influye positivamente sobre la actitud

H3: Utilidad percibida influye positivamente sobre la actitud hacia la publicidad móvil

H4: La actitud de uso influye positivamente sobre la intención de uso de la publicidad en dispositivos móviles

H5: Utilidad percibida influye positivamente sobre la intención de uso de la publicidad en dispositivos móviles 


\section{LA CONFIANZA EN LA PUBLICIDAD MÓVIL}

La confianza es una fuerza vinculante en las transacciones entre compradores y vendedores, principalmente en el comercio electrónico y móvil. Dos situaciones se presentan en este tipo de transacciones: la primera relacionada con la percepción de riesgo que presenta una de las partes, y la segunda relacionada con la asimetría de la información (Ba, 2001). Por esta razon, la percepción de confianza surge en la literatura para el caso específico de la publicidad móvil (Barakat y Sheikh, 2010) y se incluye en la investigación para desarrollar un modelo basado en el TAM.

Este tema está generando un gran interés para el ámbito de las organizaciones (Mayer, David, y Schoorman, 1995), aunque en la actualidad son varias las disciplinas que lo trabajan, tales como la sociologia, la psicologia y la economia (Ba, 2001). A comienzos de los noventa la perspectiva de confianza tomó gran valor en el comercio electrónico; la construcción de relaciones a largo plazo se convierte en el núcleo del sistema comercial (Keen y otros 1999).

La confianza es definida como la convicción que tiene un individuo de que las expectativas que posee sobre el comportamiento de la otra parte serán favorables (Gefen, 2000). Es la voluntad del individuo a ser vulnerable a las acciones del otro, basada en las expectativas de que la acción sera importante, independiente de la capacidad que se tenga para monitorear o controlar (Hans van der Heijden, Creemers y Verhagen, 2003).

También la confianza tiene una influencia directa en la intención de comportamiento del consumidor para utilizar determinada tecnologia (Cunningham, 1967) y es uno de los factores determinantes de la utilidad percibida sobre todo en un entorno móvil (Barakat y Sheikh, 2010). Gefen, Karahanna y Strauh (2003) demostraron que la utilidad percibida influye sobre la confianza. Por esta razón, para el caso de este estudio se adicionan al modelo anterior las siguientes hipotesis:

H6: La utilidad percibida influye positivamente sobre la confianza.

H7: La confianza influye positivamente sobre la intención de uso de la publicidad en dispositivos móviles.

\section{Modelo propuesto}

Para el análisis correspondiente y la validación de hipotesis referenciadas, se propone el modelo de la figura 2, para proceder a verificar, a traves del presentre estudio, las relaciones estructurales entre las variables. 
Figura 2. Modelo propuesto para validaciòn de variables

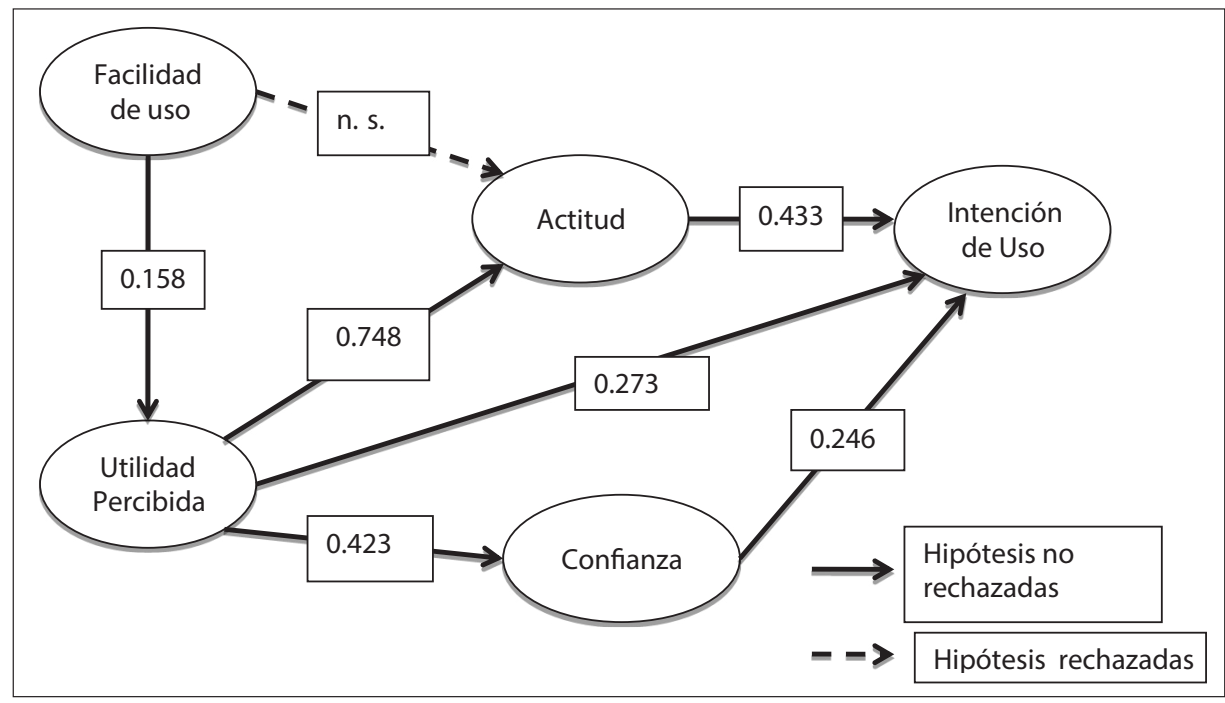

Fuente: Elaboración propia

En el modelo propuesto se ha incluido la variable confianza para validar, a traves del modelo de aceptación tecnológica (TAM), las teorias previas de la intención de uso de la publicidad en dispositivos móviles que influencian a los usuarios en Colombia.

\section{METODOLOGÍA}

La presente investigación es de corte exploratorio y descriptivo, y se abordó en dos etapas. En la primera etapa se realizó un rastreo bibliográfico tendente a determinar un estado del arte y antecedentes de estudios similares de dicho problema, configurando, además de un marco teórico, las hipótesis de la investigación. En la segunda etapa, de corte transversal simple, se buscó explicar el fenómeno objeto de estudio aplicando instrumentos de medición estructurados a una muestra seleccionada a conveniencia de 530 individuos de las principales ciudades de Colombia tales como: Medellín (incluyendo usuarios del oriente y suroeste del departamento de Antioquia), Bogotá, Cali, Barranquilla y Manizales, con una mayor participación de usuarios jóvenes y siguiendo cuotas de género acercándonos a la participación real de la población . La selección de la muestra fue por conveniencia, como numerosos escritos publicados sobre estudios similares en otros mercados (Jun y Lee (2007); Lin, Shih y She (2007); Lopez, Molina y Bouwman (2008); Altuna Konuk (2009); Chong, Chan y Ooi (2012); Casalóa, Flavián y Guinalíu (2012)). La tabla 1 recoge la ficha técnica del estudio. 
Tabla 1. Ficha técnica del estudio desarrollado

\begin{tabular}{|l|l|}
\hline Unidad muestral & Usuarios con dispositivos móviles \\
\hline Ámbito de estudio & Colombia \\
\hline Método de recogida de información & Entrevista personal \\
\hline Procedimiento de muestreo & Muestreo por conveniencia \\
\hline Tamaño muestral & 530 \\
\hline Fecha del trabajo de campo & Noviembre de 2012 \\
\hline
\end{tabular}

Fuente: elaboración propia

La tabla 2 resume las principales características de la muestra, destacándose la población con edad inferior a 35 años (84,34 \%), una proporción algo mayor de género femenino $(53,58 \%)$ y de individuos solteros $(73,21 \%)$. Predominan los individuos con formación técnica, tecnológica, pregrado incompleto y profesional $(89,43 \%)$, empleados $(35,09 \%$ y con un nivel de ingresos entre un salario mínimo (\$616.000) y \$2.500.000 (44,91\%), aclarando que sin un nivel de ingresos específico se encontró (40,19\%) que influyen de igual manera en la aceptación de la publicidad móvil (ver tabla 2). Estudios anteriores indican que las muestras compuestas en mayor proporción por jóvenes y con buen nivel educativo favorecen los estudios relacionados con el comportamiento en línea (Bigné, Ruiz, y Sanz, (2007); Netsize (2008) ; Kim, Ferrini y Rao (2008)).

Tabla 2. Caracteristicas de la muestra

\begin{tabular}{|l|c|}
\hline \multicolumn{1}{|c|}{ Edad } & $\begin{array}{c}\text { OBSERVACIONES } \\
n=530\end{array}$ \\
\hline De 12 a 18 años & $23.58 \%$ \\
\hline De 19 a 24 años & $39.43 \%$ \\
\hline De 25 a 34 años & $21.32 \%$ \\
\hline De 35 a 44 años & $9.62 \%$ \\
\hline De 45 a 55 años & $4.91 \%$ \\
\hline Mayor de 55 & $1.13 \%$ \\
\hline Género & \\
\hline Masculino & $46.42 \%$ \\
\hline Femenino & $53.58 \%$ \\
\hline Estado Civil & \\
\hline Soltero (a) & $73.21 \%$ \\
\hline Casado (a) & $13.40 \%$ \\
\hline
\end{tabular}


Bernardo Ballesteros Díaz - Juan Fernando Tavera Mesías - Daniela Castaño Serna

\begin{tabular}{|c|c|}
\hline Edad & $\begin{array}{c}\text { OBSERVACIONES } \\
n=530\end{array}$ \\
\hline Unión libre & $6.42 \%$ \\
\hline Separado (a) & $3.58 \%$ \\
\hline Divorciado (a) & $2.08 \%$ \\
\hline Viudo (a) & $1.32 \%$ \\
\hline \multicolumn{2}{|l|}{ Nivel de estudios } \\
\hline Menos de primaria & $0.75 \%$ \\
\hline Bachillerato incompleto & $2.08 \%$ \\
\hline Bachillerato completo & $19.06 \%$ \\
\hline Técnica/Tecnológica & $17.74 \%$ \\
\hline Pregrado incompleto & $34.91 \%$ \\
\hline Profesional & $17.74 \%$ \\
\hline Especialista & $4.72 \%$ \\
\hline Maestría o Doctorado & $3.02 \%$ \\
\hline \multicolumn{2}{|l|}{ Ocupación } \\
\hline Estudiante & $53.58 \%$ \\
\hline Ama de casa & $0.38 \%$ \\
\hline Trabajador independiente & $8.87 \%$ \\
\hline Empleado & $35.09 \%$ \\
\hline Desempleado & $0.75 \%$ \\
\hline Jubilado & $1.32 \%$ \\
\hline \multicolumn{2}{|l|}{ Nivel de Ingresos } \\
\hline No percibo un salario & $40.19 \%$ \\
\hline Menos de $\$ 616.000$ & $8.68 \%$ \\
\hline Entre $\$ 616.000$ y $\$ 1.000 .000$ & $21.70 \%$ \\
\hline Entre $\$ 1.000 .001$ y $\$ 1.500 .000$ & $13.21 \%$ \\
\hline Entre $\$ 1.500 .001$ y $\$ 2.500 .000$ & $10.00 \%$ \\
\hline Entre \$2.500.001 y \$3.500.000 & $3.02 \%$ \\
\hline Más de $\$ 3.500 .000$ & $3.21 \%$ \\
\hline
\end{tabular}

Fuente: elaboración propia

En la tabla 3, se presentan los ítems utilizados en el estudio y las escalas de medida, aclarando que la medición de los conceptos definidos en el modelo se hizo a través de una escala Likert de cinco opciones donde se calificó de 1 a 5 el 
grado de acuerdo o desacuerdo con las afirmaciones, siendo 1 muy en desacuerdo, 2 desacuerdo, 3 neutral, 4 de acuerdo y 5 muy de acuerdo.

Tabla 3. Escalas de medida utilizada

\begin{tabular}{|c|c|c|c|}
\hline Concepto & Código & Ítem & Fuentes \\
\hline \multirow{4}{*}{$\begin{array}{l}\text { Facilidad de uso } \\
\text { percibida }\end{array}$} & FU1 & $\begin{array}{l}\text { Aprender a utilizar un nuevo dispositivo } \\
\text { móvil es fácil para mí }\end{array}$ & \multirow{8}{*}{$\begin{array}{l}\text { (Davis (1989); Moore } \\
\text { y Benbasat (1991)) }\end{array}$} \\
\hline & FU2 & $\begin{array}{l}\text { Creo que visualizar publicidad a través de } \\
\text { mi dispositivo móvil es fácil }\end{array}$ & \\
\hline & FU3 & $\begin{array}{l}\text { No me supone ningún esfuerzo mental } \\
\text { utilizar mi dispositivo móvil }\end{array}$ & \\
\hline & FU4 & $\begin{array}{l}\text { Es fácil utilizar mi dispositivo móvil como } \\
\text { yo quiero }\end{array}$ & \\
\hline \multirow{4}{*}{ Utilidad percibida } & UPl & $\begin{array}{l}\text { Utilizar mi dispositivo móvil para recibir } \\
\text { publicidad me ofrece beneficios }\end{array}$ & \\
\hline & UP2 & $\begin{array}{l}\text { Recibir publicidad a través de mi dispositivo } \\
\text { móvil me parece muy útil }\end{array}$ & \\
\hline & UP3 & $\begin{array}{l}\text { Recibir publicidad a través de mi dispositivo } \\
\text { móvil me parece eficiente }\end{array}$ & \\
\hline & UP4 & $\begin{array}{l}\text { Recibir publicidad a través de mi dispositivo } \\
\text { móvil me parece sencillo }\end{array}$ & \\
\hline \multirow{4}{*}{$\begin{array}{l}\text { Actitud hacia } \\
\text { la recepción } \\
\text { de publicidad } \\
\text { a través de mi } \\
\text { dispositivo móvil }\end{array}$} & Al & $\begin{array}{l}\text { Recibir publicidad a través de mi dispositivo } \\
\text { móvil es una idea que me gusta }\end{array}$ & \multirow{4}{*}{ (Taylor y Todd (1995)) } \\
\hline & A2 & $\begin{array}{l}\text { Recibir publicidad a través de mi dispositivo } \\
\text { móvil me parece una idea inteligente }\end{array}$ & \\
\hline & A3 & $\begin{array}{l}\text { Recibir publicidad a través de mi dispositivo } \\
\text { móvil es una buena idea }\end{array}$ & \\
\hline & A4 & $\begin{array}{l}\text { Recibir publicidad a través de mi dispositivo } \\
\text { móvil es una idea positiva }\end{array}$ & \\
\hline \multirow{3}{*}{$\begin{array}{l}\text { Confianza } \\
\text { Percibida }\end{array}$} & $\mathrm{COl}$ & $\begin{array}{l}\text { Creo que la publicidad que recibo a través } \\
\text { de mi dispositivo móvil es confiable }\end{array}$ & \multirow{3}{*}{$\begin{array}{l}\text { (Kim, Ferrini y Rao, } \\
\text { (2008); Kim, Chung, y } \\
\text { Lee (2011)) }\end{array}$} \\
\hline & $\mathrm{CO} 2$ & $\begin{array}{l}\text { Creo que mi dispositivo móvil es confiable } \\
\text { para proteger mi información personal }\end{array}$ & \\
\hline & $\mathrm{CO} 3$ & $\begin{array}{l}\text { Creo que la publicidad que recibo a través } \\
\text { de mi dispositivo móvil es honesta }\end{array}$ & \\
\hline \multirow{3}{*}{$\begin{array}{l}\text { Intención de uso } \\
\text { Publicidad móvil }\end{array}$} & IU1 & $\begin{array}{l}\text { Tengo la intención de aceptar publicidad a } \\
\text { través de mi dispositivo móvil }\end{array}$ & \multirow{3}{*}{$\begin{array}{l}\text { (Taylor y Todd (1995); } \\
\text { Coyle y Thorson } \\
\text { (2001) y Kim y Biocca } \\
\text { (1997) }\end{array}$} \\
\hline & IU2 & $\begin{array}{l}\text { Pienso que recibiré publicidad a través de } \\
\text { mi dispositivo móvil en los próximos meses }\end{array}$ & \\
\hline & IU3 & $\begin{array}{l}\text { Recomendaré a otros a aceptar publicidad } \\
\text { a través de sus dispositivos móviles }\end{array}$ & \\
\hline
\end{tabular}

Fuente: elaboración propia 


\section{RESULTADOS Y DISCUSIÓN}

Para la comprobación de validez de escalas de medida utilizadas se realizó, a través del aplicativo EOS $6.2^{1}$, un análisis factorial confirmatorio.

Dada la presencia de curtosis multivariante, el análisis se desarrolló utilizando indicadores robustos que permiten corregir los problemas de anormalidad de los datos presentes.

Se recurrió a la eliminación de los indicadores UP4 y IU2, porque las cargas factoriales eran inferiores a 0,6, para lograr convergencia del modelo de acuerdo con Bagozzi y Yi (1988). Ajustado el modelo de medida se evidenció que las cargas factoriales estandarizadas, además de significativas, superaron 0,6. De acuerdo con lo presentado en la tabla 4, el promedio de las cargas de los indicadores en relación con cada factor fue superior a 0,7, (Hair y otros (2001)).

Tabla 4. Análisis confirmatorio de la validez convergente

\begin{tabular}{|c|c|c|c|c|}
\hline Constructo & Ítem & $\begin{array}{c}\text { Cargas factoriales } \\
\text { estandarizadas }\end{array}$ & $\begin{array}{c}\text { Promedio cargas factoriales } \\
\text { estandarizadas }\end{array}$ & $\begin{array}{l}\text { Valort } \\
\text { robusta }\end{array}$ \\
\hline \multirow{4}{*}{ Facilidad de Uso (FU) } & FU1 & 0,739 & \multirow{4}{*}{0,7430} & 16,748 \\
\hline & FU2 & 0,610 & & 13,643 \\
\hline & FU3 & 0,822 & & 19,845 \\
\hline & FU4 & 0,801 & & 17,388 \\
\hline \multirow{3}{*}{ Utilidad Percibida (UP) } & UP1 & 0,842 & \multirow{3}{*}{0,892} & 27,163 \\
\hline & UP2 & 0,941 & & 39.350 \\
\hline & UP3 & 0,894 & & 30,761 \\
\hline \multirow{4}{*}{ Actitud (A) } & $\mathrm{A} 1$ & 0,805 & \multirow{4}{*}{0,877} & 24,006 \\
\hline & A2 & 0,864 & & 29,308 \\
\hline & A3 & 0,931 & & 36,674 \\
\hline & A4 & 0,909 & & 33,914 \\
\hline \multirow{3}{*}{ Confianza (CO) } & $\mathrm{COl}$ & 0,871 & \multirow{3}{*}{0,8397} & 26,925 \\
\hline & $\mathrm{CO} 2$ & 0,735 & & 20,255 \\
\hline & $\mathrm{CO} 3$ & 0,913 & & 26,270 \\
\hline \multirow[t]{2}{*}{ Intención de Uso (IU) } & IU1 & 0,900 & \multirow{2}{*}{0,887} & 28,748 \\
\hline & IU3 & 0,874 & & 27,754 \\
\hline
\end{tabular}

Fuente: elaboración propia

EOS 6.2: Structural Equation Program, Multivariate Software. Software para modelar ecuaciones estructurales versión 6.2. Programa que facilita la aplicación de la estadística avanzada para modelos multivariados en procesos de investigación. Para mayores detalles sobre este software consultar Blenter (2006). 
El análisis de la validez discriminante se ejecutó mediante (a) la comprobación de que el intervalo de confianza en la estimación de la correlación entre cada par de factores no contuviera el valor 1 (Anderson y Gerbing, 1988), y (b) que la varianza promedio extraída para cada factor fuera mayor que el cuadrado de la correlación entre cada par de factores (Fornell y Larcker, 1981). La tabla 5 evidencia que todos los casos cumplen con la validez discriminante, y la tabla 6 muestra los constructos que obtuvieron un Alfa de Cronbach superior a 0,7, índices de fiabilidad compuesta superiores a 0,6, e índices de varianza extraída superiores a 0,5.

Tabla 5. Validez discriminante del modelo de medida

\begin{tabular}{|l|c|c|c|c|c|}
\hline & FU & UP & A & CO & IU \\
\hline FU & 0,748 & 0,157 & 0,151 & 0,112 & 0,099 \\
\hline UP & {$[0,251 ; 0,063]$} & 0,893 & 0,748 & 0,408 & 0,691 \\
\hline A & {$[0,245 ; 0,057]$} & {$[0,791 ; 0,705]$} & 0,879 & 0,448 & 0,737 \\
\hline CO & {$[0,208 ; 0,016]$} & {$[0,486 ; 0,330]$} & {$[0,522 ; 0,374]$} & 0,843 & 0,543 \\
\hline IU & {$[0,197 ; 0,001]$} & {$[0,746 ; 0,636]$} & {$[0,786 ; 0,688]$} & {$[0,614 ; 0,472]$} & 0,887 \\
\hline
\end{tabular}

${ }^{* *} \mathrm{p}<, 01 ;{ }^{*} \mathrm{p}<, 05$. Debajo de la diagonal: intervalo de confianza para la correlación entre factores. Diagonal: raíz cuadrada de la varianza extraída. Sobre la diagonal: correlación estimada entre los factores.

Fuente: elaboración propia

Tabla 6. Índices de fiabilidad

\begin{tabular}{|l|c|c|c|}
\hline \multicolumn{1}{|c|}{ Constructos } & Alfa de Cronbach & $\begin{array}{c}\text { Índice de Fiabilidad } \\
\text { Compuesta (IFC) }\end{array}$ & $\begin{array}{c}\text { Índice de Varianza } \\
\text { Extraída (IVE) }\end{array}$ \\
\hline Facilidad de uso (FU) & 0,830 & 0,627 & 0,559 \\
\hline Utilidad percibida (UP) & 0,881 & 0,815 & 0,798 \\
\hline Actitud (A) & 0.928 & 0,794 & 0,772 \\
\hline Confianza CO & 0,873 & 0,744 & 0,711 \\
\hline Intención de uso (IU) & 0,734 & 0,806 & 0,787 \\
\hline
\end{tabular}

Fuente: elaboración propia

Dadas las pruebas realizadas, las propiedades psicométricas del instrumento de medida son satisfactorias de acuerdo con los indicadores de fiabilidad, y la validez convergente y discriminante. 


\section{Análisis de resultados y contraste de hipótesis}

Fue utilizado el método de verosimilitud robusto para validar las escalas de medida para contrastar, analizar e interpretar las hipótesis planteadas.

El modelo estructural propuesto y sus hipótesis fue especificado, realizando su estimación, interpretación y evaluación de ajuste (Kaplan, 2000).

La tabla 7 presenta los parámetros estimados para la confirmación o rechazo de las hipótesis propuestas y describe los indicadores observados en el modelo.

Tabla 7. Contraste de hipótesis

\begin{tabular}{|c|c|c|}
\hline Hipótesis & $\begin{array}{c}\text { Carga } \\
\text { estandarizada }\end{array}$ & $\begin{array}{l}\text { Valor } t \\
\text { robusto }\end{array}$ \\
\hline $\begin{array}{l}\text { H1: Facilidad de uso percibida influye positivamente } \\
\text { sobre la utilidad percibida }\end{array}$ & $0,158 * * *$ & 3,468 \\
\hline $\begin{array}{l}\text { H2: Facilidad de uso percibida influye positivamente } \\
\text { sobre la actitud }\end{array}$ & n. s. & \\
\hline $\begin{array}{l}\text { H3: Utilidad percibida influye positivamente sobre la } \\
\text { actitud hacia la publicidad móvil }\end{array}$ & $0,748 *, * *$ & 15,262 \\
\hline $\begin{array}{l}\text { H4: La actitud de uso influye positivamente sobre la } \\
\text { intención de uso de la publicidad en dispositivos } \\
\text { móviles }\end{array}$ & $0,433 *, * *$ & 6,696 \\
\hline $\begin{array}{l}\text { H5: Utilidad percibida influye positivamente sobre la } \\
\text { Intención de uso de la publicidad en dispositivos } \\
\text { móviles }\end{array}$ & $0,273 *, * *$ & 3,957 \\
\hline $\begin{array}{l}\text { H6: La utilidad percibida influye positivamente sobre la } \\
\text { confianza }\end{array}$ & $0,423 *, * *$ & 8,155 \\
\hline $\begin{array}{l}\text { H7: La confianza influye positivamente sobre la intención } \\
\text { de uso de la publicidad en dispositivos móviles }\end{array}$ & $0,246^{*}, * *$ & 5,672 \\
\hline \multicolumn{3}{|c|}{$\begin{array}{l}\text { S-B X } X^{2}=210,6267 ; \text { g. } 1 .=97 ; \mathrm{p}<0,01 ; \text { BBNFI }=0,962 ; \mathrm{BBNNFI}=0,974 ; \mathrm{CFI}=0,979 ; \mathrm{IFI}=0,979 ; \\
\text { RMSEA }=0,047[0,038-0,056] ;{ }^{* *} \mathrm{p}<, 01 ;{ }^{*} \mathrm{p}<, 05 ; \text { n. s.: no soportada }\end{array}$} \\
\hline
\end{tabular}

Fuente: elaboración propia

Según los resultados obtenidos, después de validar el modelo, podemos afirmar que la facilidad de uso influye de manera directa sobre la utilidad percibida frente a la publicidad en dispositivos móviles ( $\mathrm{Hl}$ aceptada; $\mathrm{B}=0.158 ; \mathrm{p}<0,05$ ) y a su vez no influye en la actitud (H2 rechazada). Por otra parte, la utilidad percibida tiene influencia sobre la actitud ( $\mathrm{H} 3$ aceptada; $\mathrm{B}=0.748 ; \mathrm{p}<0,01$ ), así como sobre la intención de uso ( $\mathrm{H} 5$ aceptada; $\mathrm{B}=0.273 ; \mathrm{p}<0,01$ ) y la confianza (H6 aceptada; $\mathrm{B}=0.423 ; \mathrm{p}<0,01$ ). 
Se demuestra que las variables utilizadas del modelo TAM fueron aceptadas, excepto la relación de la facilidad de uso con la actitud; sin embargo, se ratifica que esta última sí tiene una influencia directa sobre la intención de los usuarios para aceptar la publicidad en dispositivos móviles ( $\mathrm{H} 4$ aceptada; $\mathrm{B}=0.433$; $\mathrm{p}<0,01)$.

La variable confianza, como nuevo concepto adicionado al modelo TAM, influye de manera directa en la intención de uso de los usuarios de publicidad en dispositivos móviles (H7 aceptada; $\mathrm{B}=0.246 ; \mathrm{p}<0,01$ ).

La figura 3 ilustra el cumplimiento de las hipótesis propuestas con las respectivas cargas.

Figura 3. Cumplimiento de hipótesis del modelo propuesto

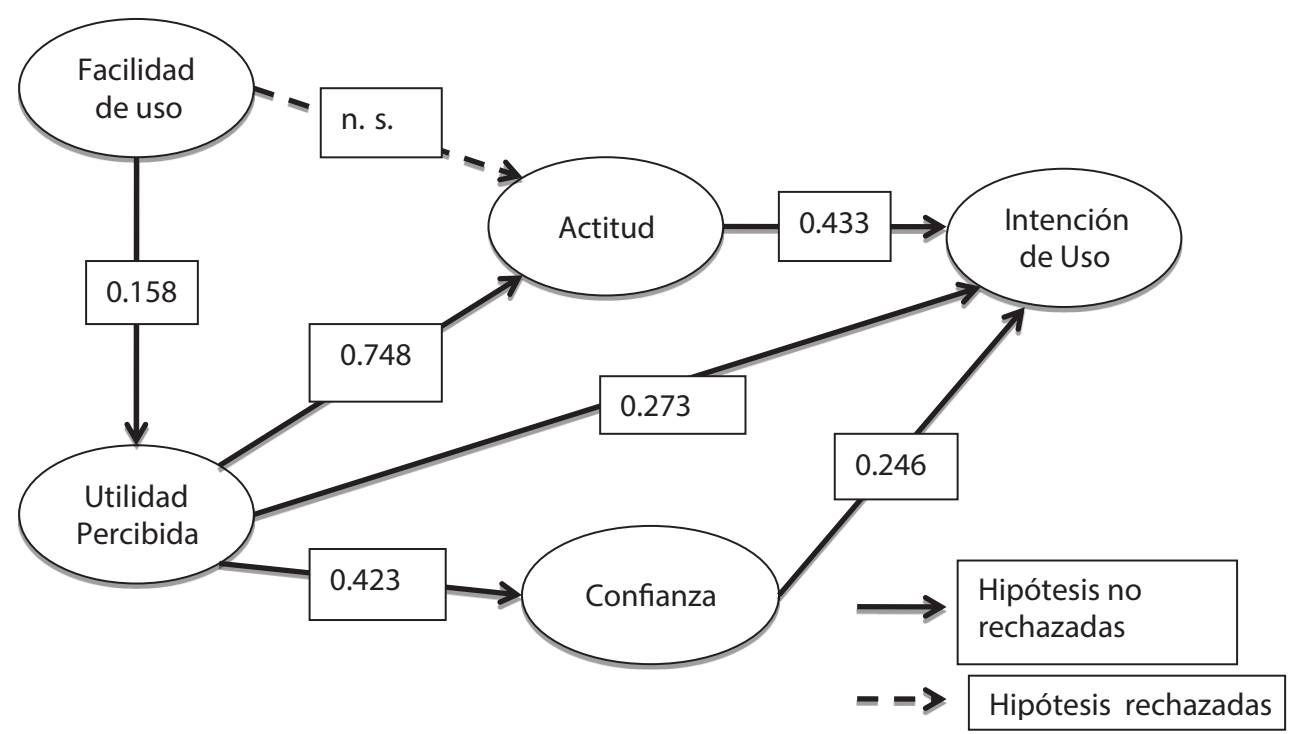

Fuente: elaboración propia

La tabla 8 presenta los resultados del efecto total de los conceptos involucrados en el modelo, respecto de la intención de uso de la publicidad móvil. Una vez analizadas las cargas estandarizadas de las relaciones entre conceptos con efecto directo e indirecto, se evidencia que la utilidad percibida es el factor que mayor efecto tiene, sobre la intención de uso de la publicidad en dispositivos móviles para los consumidores colombianos. 
Tabla 8. Efecto de los constructos sobre la intención de uso

\begin{tabular}{|l|c|c|c|}
\hline \multicolumn{1}{|c|}{ Variable } & Efecto directo & Efecto indirecto & Efecto total \\
\hline Facilidad de uso (FU) & 0 & 0,110 & 0,110 \\
\hline Utilidad percibida (UP) & 0,273 & 0,323 & 0,596 \\
\hline Actitud (A) & 0,433 & 0 & 0,433 \\
\hline Confianza (CO) & 0,246 & 0 & 0,246 \\
\hline
\end{tabular}

Fuente: elaboración propia

\section{CONCLUSIONES}

Conocer acerca del comportamiento del consumidor para orientar la gestión de marketing de las organizaciones y para los estudios académicos, siempre ha merecido atención por su complejidad, importancia y utilidad. Al respecto se encuentran desde estudios generales sobre influencia del marketing móvil en la toma de decisiones de los clientes (Shankar \& Balasubramanian, 2009), hasta investigaciones específicas como el caso de la comunicación móvil a través servicios de mensajes cortos (SMS) (Scharl, Dickinger y Murphy (2005)).

La investigación aporta a la literatura sobre este tema que ha sido estudiado en otros contextos utilizando el modelo de aceptación tecnológica, con consumidores de los Estados Unidos (Jun y Lee (2007); China (Zhang y Mao (2008) y Pakistan (Sultan, Rohm y Gao, (2009)); para el caso de Colombia y de Latinoamérica existe un vacío.

Respecto a la metodología, se ratifica el ajuste del modelo utilizado con los datos obtenidos de la muestra, donde se identifica una fuerte influencia de la utilidad percibida sobre la actitud y de esta sobre la intención de uso en la aceptación de la publicidad en dispositivos móviles. La facilidad de uso influye de manera indirecta, a través de la utilidad percibida, sobre la aceptación e intención de uso de la publicidad, pero no se encuentra ninguna influencia directa sobre la actitud, lo que coincide con otros estudios realizados del tema de aceptación de tecnologías (Ruiz, Sanz y Tavera (2010); Tavera, Sánchez, y Ballesteros (2011)).

Por otra parte, se encuentra una influencia directa de la utilidad percibida sobre la confianza y a través de esta última variable, adicionada en la presente investigación al modelo original TAM, una influencia indirecta sobre la intención de uso y aceptación de la publicidad en dispositivos móviles para el caso del consumidor en Colombia. La confianza, como variable antecedente de la intención de uso, ha sido ratificada en otros estudios e investigaciones efectuadas (Suh y Han (2003); Palvia (2009); Hans van der Heijden, Creemers y Verhagen (2003); Barakat y Sheikh (2010)). 
Al analizar las cargas estandarizadas de las relaciones entre conceptos, la utilidad percibida tiene un mayor efecto total sobre la intención de uso de la publicidad en dispositivos móviles para los consumidores colombianos, ratificando lo planteado por Davis en el modelo original del TAM.

Los resultados encontrados permiten, a los responsables de la gestión de marketing y a la gerencia de las organizaciones, interpretar de manera acertada los comportamientos de los consumidores frente a estrategias que pueden ser diseñadas a la medida de los mercados y en función de los objetivos empresariales, teniendo presentes los condicionantes a la hora de tomar decisiones de compra o utilización de servicios en el marco de la oferta y la demanda.

El contar con información de la aceptación tecnológica de la publicidad a través de dispositivos móviles por parte del consumidor colombiano y la relación de variables que le afectan permite a las organizaciones realizar un despliegue estratégico hacia los consumidores que, además de permitir la optimización de recursos por su especificidad, logre un impacto favorable en la aceptación y respuesta frente a la oferta.

A futuro es posible utilizar nuevas variables que tengan relación con aspectos de personalización y comportamiento del consumidor frente a la aceptación de tecnologías, utilizando el modelo TAM que ha sido validado y reconocido en este tipo de estudios para futuras investigaciones. En la revisión literaria se encontró que varios estudios relacionados utilizan variables tales como interactividad, irritabilidad, aceptación del riesgo, capacidad de respuesta, ubicuidad, entre otras.

Las principales limitaciones del proyecto de investigación son: primero, la muestra es no probabilística a conveniencia; por lo tanto, no se podrían generalizar las conclusiones en todos los ámbitos en los cuales se abordó el estudio, y segundo, el modelo propuesto solo llega hasta el concepto intención de uso; no se midió de manera directa el uso ya que es difícil encontrar una alta proporción de usuarios de actividades publicitarias a través de dispositivos móviles. Finalmente, se recomienda validar el TAM hasta el uso con una muestra de usuarios de este tipo de servicios.

\section{BIBLIOGRAFÍA}

Ajzen, I., y Fishbein, M. (1977). Attitude-behaviour relations: A theorical analysis and review of empirical research. En: Psycological Bulletin, Vol. 84, No. 5, p. 888-819.

Altuna, O. K., y Konuk, F. A. (2009).Understanding Constumer Attitudes Toward Mobile Advertising and Its Impact on Consumers' Behavioral Intentions: a Cross-Market Comparison of United States and Turkish Consumers. En: International Journal of Mobile Marketing, Vol. 
4, No. 2, p. 43-51.

Anderson, J. C., y Gerbing, D. W. (1988). Structural equation modeling in practice: A review and recommended two-step approach. En: Psychological bulletin, Vol. 103, No. 3, p. 411 423.

Ba, S. (2001). Establishing online trust through a community. En: Decision Support Systems, Vol. 31, p. 323-336.

Bagozzi, R. P., y Yi, Y. (1988). On the evaluation of structural equation models. En: Journal of Academy of Marketing Science, Vol. 16, No. 1, p. 74-94.

Bamba, F., y Barnes, S. J. (2007). SMS advertising, permission and the consumer: a study. En Business Process Management Journal, Vol. 13, No. 6, p.815-829.

Barakat, s., y Sheikh, A. E. (2010). Trust and User Acceptance of Mobile advertising. En: European, Mediterranean $\&$ Middle Eastern Conference on Information Systems, p. 1-9.

Bart, Y., Stephen, A. T., y Sarvary, M. (2014). Which products are best suited to mobile advertising? A field study of mobile display advertising effects on consumer attitudes and intentions. En: Journal of Marketing Research, Vol. 51, No. 3, p. 270-285.

Bigné, E., Ruiz, C., y Sanz, S. (2007). Key Drivers of Mobile commerce Adoption.An Exploratory Study of Spanish Mobile Users. En: Journal of theorical and Applied Electronic Commerce Research, Vol. 2, No. 2, p. 48.

Bina, M., Karaiskos, D., y Giaglis, G. M. (2007). Motives and Barriers Affecting the use of Mobile Data Services. En: Proceedings of the IEEE International Conference on Mobile Business (ICMB 2007).

Blenter, P. M. (2006). EQS structural equations program. Universidad de California, Los Ángeles, 418p.

Casalóa, L. V., Flavián, C. y Guinalíu, M. (2012). Redes sociales virtuales desarrolladas por organizaciones empresariales: antecedentes de la intención de participación del consumidor. En: Cuadernos de Economía y Dirección de la Empresa, Vol. 15, No. 1, p. 42-51.

Chong, A., Chan, F., y Ooi, K. (2012). Predicting consumer decisions to adopt mobile commerce: Cross country empirical examination between China and Malaysia. En: Decision Support Systems, Vol. 53, No. 1, p. 34-43.

Coyle, J., y Thorson, R. (2001). The effects of progressive levels of interactivity and vividness in Web marketing sites. En: Journal of advertising, Vol. 30, No. 3, p. 65.

Cunningham, S. (1967). The major dimensions of perceived risk. En: Cox, D. (Ed.), Risk taking and information handling in consumer behavior. Cambridge, $\overline{\mathrm{MA}}$ : Harvard University Press.

Davis, F. D. (1989). Perceived usefulness, perceived ease of use, and user acceptance of information technologies. En MIS Quarterly, Vol. 13, No. 3, p. 21.

Davis, F. D., Bagozzi, R. P., y Warshaw, P. R. (1989). User Acceptance of Computer Technology: A Comparison of two theoretical Models. En: Management Science, Vol.35, No. 8, p. 982-1003. 
Fishbein, M., y Ajzen, I. (1975). Belief, attitude, intention, and behavior: An introduction to theory an research. Reading Mass. Ed. Addison-Wesley.

Fornell, C., y Larcker, D. F. (1981). Evaluating structural equation models with unobservable variables and measurement error. En: Journal of marketing research, Vol. 18, No. 1, p. 39-50.

Gefen, D. (2000).E-commerce: the role of familiarity and trust. En: Omega, Vol. 28, p. 725-737.

Gefen, D., Karahanna, E., y Straub, D. W. (2003). Inexperience and experience with online stores: The importance of TAM and trust. En: IEEE Transactions on Engineering Management, Vol. 50, No. 3, p. 307-322.

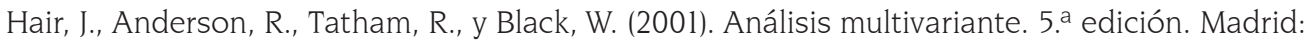
Ed. Prentice Hall Iberia.799p.

Hans van der Heijden, Creemers, M., y Verhagen, T. (2003). Understanding online purchase intentions. En European Journal of Information Systems, Vol. 12, p. 41-48.

He, D., y Lu, Y. (2007). Consumers Perceptions and Acceptances towards Mobile Advertising: An Empirical Study in China. En: Wireless Communications, Networking and Mobile Computing. WiCom 2007. International Conference on (p. 3775-3778). IEEE.

Hsiao, W. H., y Chang, T. S. (2014). Understanding consumers' continuance intention towards mobile advertising: a theoretical framework and empirical study. En: Behaviour $\mathcal{E}$ Information Technology, Vol. 33, No. 7, p. 730-742.

IAB Colombia. (2015): Reporte sobre la inversión en medios digitales en Colombia. [En línea]. Bogota: Pricewterhousecoopers. [13 agosto de 2015].

Jun, J. W., y Lee, S. (2007). Mobile media use and its impact on consumer attitudes toward mobile advertising. En: International Journal of Mobile Marketing, Vol.2, No 1, p. 50.

Jun, J., y Lee, T. (2007). Contextual perceived value? Investigating the role of contextual Marketing for customer relationship management in a mobile commerce context. En: Business Process Management, Vol. 2, No. 1, p. 50-58.

Kaplan, D. (2000). Structural Equation Modeling: Foundations and Extensions. Thousand Oaks. Ed. SAGE Publications.

Keen, P., Ballance, G., Chan, S., y Schrump, S. (1999). Electronic Commerce Relationships: Trust by Design. Englewood Cliffs NJ, Prentice-Hall.

Kim, D. J., Ferrini, D. L., y Rao, H. R. (2008). A trust-based consumer decision-making model in electronic commerce: The role of trust, perceived risk, and their antecedents. En Decision Support System, Vol.44, No.2, p. 544-564.

Kim, M. -J., Chung, N., y Lee, C. -K. (2011). The effect of perceived trust on electronic commerce: Shopping online for tourism products and services in South Korea. En: Tourism Management, Vol. 32, No. 2, p. 256-265.

Kim, T., y Biocca, F. (1997). Telepresence via television: two dimensions of telepresence may have diferent connections to memory and persuasión. [En línea]]. Consultado en Octubre 2012. 
Lamb, C. W., Hair, J. F., y Mcdaniel, C. (1998). Marketing (4th ed.). México: Ed. International Thomson Editores.

Lin, C. H., Shih, H. Y., y Sher, P. J. (2007). Integrating Technology Readiness into Technology Acceptance: The TRAM Model. En: Psychology E Marketing , Vol. 24, N. ${ }^{\circ}$ 7, p. 641-657.

López, C., Molina, F. J., y Bouwman, H. (2008). An assessment of advanced mobile services acceptance: Contributions from TAM and diffusion theory models. En: Information $\mathcal{E}$ Management, Vol. 45, No. 6, p. 359-364.

Mayer, R. C., David, F. D., y Schoorman, F. D. (1995). An integrative model of organizational trust. En Academy of Management Review, Vol. 20, No. 3, p. 709-734.

MTIC- Ministerio de Tecnologías de la información y las Comunicaciones de Colombia (2015). Boletín Trimestral de las TIC, cifras primer trimestre 2015. [En línea] Bogotá, julio 2015. [Consultado el 13 agosto de 2015].

Molina, F. J., López, C., y Soto, P. (2012). Interactions effects of media and message on perceived complexity, risk and trust of innovative products. En: European Management Journal, Vol. 30, p. 577-587.

Moore, G., y Benbasat, I. (1991). Development of an instrument to measure the perception of adopting an information technology innovation. En: Information Systems Research, Vol. 2, No. 3, p. 199-222.

Muk, A., y Chung, C. (2015). Applying the technology acceptance model in a two-country study of SMS advertising. En: Journal of Business Research, Vol. 68, No. 1, p. 1-6.

Netsize (2008): The Netsize guide 2008. [En línea] Información corporative de NETSIZE - Gemalto Corporation.

Palvia, P. (2009). The role of trust in e-commerce relational exchange: A unified model. En Information E Management, Vol.46, No. 4, pp. 213-220.

Ruiz, C. M., Sanz, S. B., y Tavera, J. F. (2010). A comparative study of mobile messaging services acceptance to participate in television. En: Programmes Journal of Service Management, Vol. 21, No. 1, p. 69-102.

Sanz-Blas, S., Ruiz-Mafé, C., y Martí-Parreño, J. (2015). Message-driven factors influencing opening and forwarding of mobile advertising messages. En: International Journal of Mobile Communications, Vol. 13, No. 4, p. 339-357.

Scharl, A., Dickinger, A., y Murphy, J. (2005). Diffusion and success factors of mobile marketing. En: Electronic Commerce Research and applications, Vol. 4, p.159-173.

Shankar, V., y Balasubramanian, S. (2009). Mobile Marketing: A synthesis and prognosis. En: Journal of Interactive, Vol. 23, No. 2, p. 118-129.

Suh, B., y Han, I. (2003). The impact of trust and perception of security control on the acceptance of electronic commerce. En: International Journal of Eletronic Commerce, Vol. 7, No. 3, p. $135-161$. 
Sultan, F., Rohm, A. J., y Gao, T. T. (2009). Factors Influencing Consumer Acceptance of Mobile Marketing: A Two-Country Study of Youth Markets. En: Journal of Interactive Marketing, Vol. 23, No. 4, p. 308-320.

Tavera, J. F., Sánchez, J. C., y Ballesteros, B. (2011). Aceptación del e-commerce en Colombia: un estudio para la ciudad de Medellín. En: Revista Facultad de Ciencias Económicas: Investigación y Reflexión, Vol. 19, No. 2, p. $\overline{5-7}$.

Taylor, S., y Todd, P. (1995). Understanding information technology usage: A test of competing models. En: Information Systems Research, Vol. 6, No. 2, p. 144.

Tseng, F. M., y Lo, H. Y. (2011). Antecedents of consumers' intentions to upgrade their mobile phones. En: Telecommunications Policy, Vol. 35, No.1, p. 74-86

Venkatesh, V. y Davis, F.D. (2000). A Theoretical Extension of the Technology Acceptance Model: Four Longitudinal Field Studies. En: Management Science, Vol. 46, p. 186-204.

Venkatesh, V., y Bala, H. (2013). TAM 3: Advancing the technology acceptance model with a focus on interventions. Manuscript in preparation.

Yang, C. H., Liu, H., y Zhou, L. (2012). Predicting young Chinese consumers' mobile viral attitudes, intents and behavior. En: Asia Pacific Journal of Marketing and Logistics, Vol. 24, No. 1, p. 59-77.

Yuan, S., y Tsao, Y. W. (2003). A recomendation mechanism for contextualized mobile adversiting. En: Expert System with Applications, Vol. 24, p. 399-414.

Zhang, J., y Mao, E. (2008). Understanding the Acceptance of Mobile SMS Advertising among Young Chinese Consumers. En: Psychology \& Marketing, Vol.25, No. 8, p. 787-805. 
